\title{
Die USA nach der Wahl
}

Nachdem Joe Biden die Präsidentschaftswahlen in den USA für sich entscheiden konnte, stellt sich die Frage, wie sich die transatlantischen Beziehungen zwischen den USA und der Europäischen Union zukünftig entwickeln werden. Das Verhältnis zwischen der USAdministration unter Donald Trump und der EU war durch gegenseitiges Misstrauen geprägt, das sich in verbalen Attacken und konkret in Strafzöllen ausgewirkt hat. Nun schlägt Joe Biden versöhnlichere Töne an, weshalb die EU auf einen Neustart nicht nur der transatlantischen Handelsbeziehungen hoffen darf. Die Autorinnen und Autoren analysieren, wie sich die USA in den Jahren der Trump-Administration wirtschaftlich und gesellschaftlich entwickelt haben und geben einen Ausblick auf die zukünftigen Herausforderungen und Potenziale der transatlantischen Beziehungen.

\section{Transatlantische Handelspolitik vor, während und nach Trump}

Gabriel Felbermayr, Institut für Weltwirtschaft (IfW) Kiel; Christian-Albrechts-Universität Kiel.

\section{Build back better: Neustart transatlantischer Handelsbeziehungen?}

Stormy-Annika Mildner, Bundesverband der deutschen Industrie (BDI), Berlin; Hertie School of Governance, Berlin. Elisabeth Allmendinger, Bundesverband der deutschen Industrie (BDI), Berlin.

\section{Der amerikanische Traum ist ausgeträumt}

Michael Hüther, Institut der deutschen Wirtschaft (IW) Köln; European Business School, Oestrich-Winkel.

\section{Der amerikanische gordische Knoten}

John Komlos, ehemals Ludwig-Maximilians-Universität München.

Hermann Schubert, International School of Management, Stuttgart. 